UDK 78(437)"312" : 8.08511

\title{
SPRACHE IN DER TSCHECHISCHEN NEUEN MUSIK
}

\author{
Jarmila Dou bravová (Praga)
}

Vokalwerke der tschechischen Neuen Musik werden durch mehrere Züge charakterisiert. Obwohl es in der tschechischen Neuen Musik der sechziger Jahre keinen Widerspruch zwischen der Vokal-und Instrumentalmusik gibt, ist ihr Anfang überwiegend mit der Instrumentalmusik verbunden, während die Vokalmusik sich erst von der zweiten Hälfte der sechziger Jahre an entwickelte. Diese nicht nur tschechische Tatsache ist durch den projizierten Charakter der neuen Kompositionsverfahren bedingt. Sie hat ihre Folgerung in dem experimentalen Charakter der neuen Vokalmusik, einschließlich der Suche nach einer neuen Beziehung zwischen der Sprache und der Musik.

Während die Entwicklung der Vokalmusik im 19. Jh. und in der ersten Hälfte des 20. Jhs. - mit der Ausnahme der Wiener Schule - auf eine Art der Vertonung ausgerichtet wurde, die auf einem Widerspruch zwischen dem Text und der Musik, und auf der Bemühung diesen Widerspruch $\mathrm{zu}$ überwinden, begründet wurde, fand die Neue Musik die Möglichkeit zur Beseitigung dieses Widerspruchs, indem sie die Klangschichte der Sprache als musikalisches Material benützte. Eine Anregung gab ihr auch die experimentale Poesie (H. Chopin, F. Dufrêne, bei uns J. Hiršal und andere). Gleichzeitig wurde eine Entdeckung der Wiener Schule weiterentwickelt, nämlich bewußte Verneigung des Widerspruchs zwischen der Sprache und der Musik, indem die Vokalmusik den Gesetzen der neuen Kompositionsverfahren untergeordnet wurde.

Wenn die Unterstellung der vokalen Äußerung den Gesetzmäßigkeiten der projektiven Kompositionstechniken typisch für den Anfang der tschechischen vokalen Neuen Musik der sechziger Jahre war, so wurde die weitere Entwicklung mit der Ausnützung der Sprache als Baumaterial der Vokalmusik verbunden. Das Anfangsstadium der tschechischen vokalen Neuen Musik kann hier durch folgende Kompositionen vorgestellt werden: 3 Sonnets from Shakespeare auf englischen Text, eine serielle Komposition von Zbyněk Vostrák (1964, Urauff-1964 Roma, Schallplatte-Supraphon 1971), die Freske für gemischten Chor und Flöte "Die Mutter" von Marek Kopelent (1964, Urauff-1965 Warszawa, Druck-Panton 1969, Schpl-Panton 1972) 
und "Inventionen für gemischten Chor" von Jindřich Feld (1966, SchplPanton 1972).

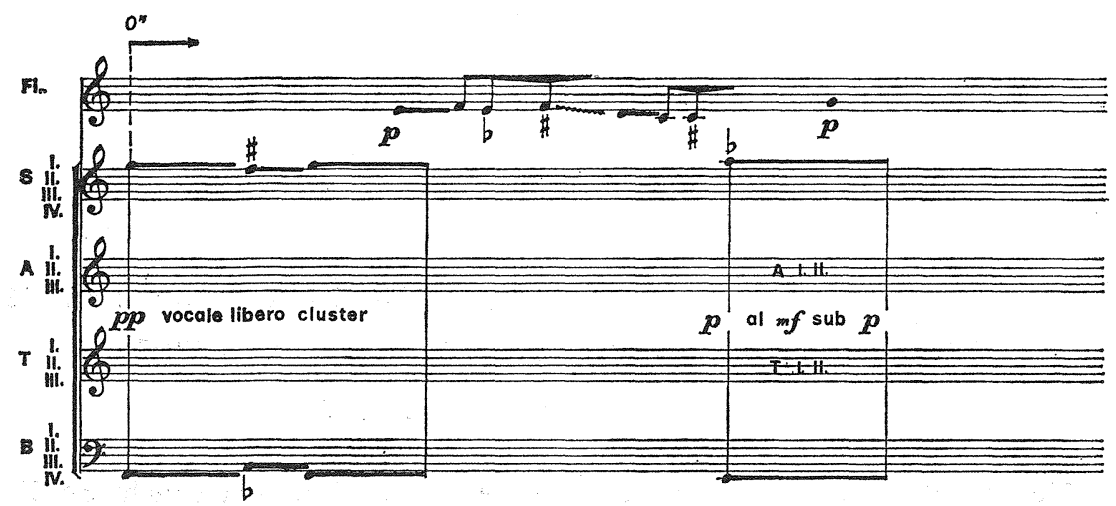

In der weiteren Entwicklung wurde die Doppelbedeutung der Sprache - als Begriff und Klang - verwendet. Die Tendenz zur maximalen Ausnützung der begrifflichen seite der Sprache machte sich auf mehrere Weisen bemerkbar, von denen in diesem Zusammenhang zwei zu erwähnen sind: erstens die Stellung des Wortes vor die Musik und zweitens die Ausnuitzung verschiedener literarischen Stile. Ein eindruckvolles Beispiel der ersten Richtung bietet die Komposition Svatopluk Havelkas "Der Schaum» (1965, Urauff-1966 Praha, Schpl-Panton 1966). Sie entstand als Musik zum Gedicht von H. M. Enzensberger. Dieses Werk, das keine sinfonische Dichtung ist und auch keine sein will, ist auf Variationsverwandlungen eines Leitmotivs von dem Hintergrund von reich veränderlichen Timbre-Strukturen gebaut. Die 7. Sinfonie Miloslav Kabeláčs (1967, Urauff-1968 Praha) entstand auf den Text vom Komponisten nach dem Evangelium und der Offenbarung des hl. Johannes und ist für Orchester und einen Sprecher bestimmt. Der Wortteil konzentriert sich auf wenige bedeutungsvolle Worte: Ewigkeit, Anfang, Wort, Leben, Geheimnis u. a., von denen der Komponist mittels der Kombinierung, der klanglichen Interpunktion und selbstverständlich der Musik eine Polemik mit der biblischen Interpretation entfaltet. In der kompositorischen Hinsicht wird dieses Werk ähnlich wie alle Kompositionen Kabeláčs durch eigenartige Technik gekennzeichnet, die auf rationell ausgewählten Material und vorbestimmter zeitlicher Organisation des Werkes begründet ist.

Auf der Ausnützung des stilistischen Charakters der Sprache ist die Komposition von Alois Simandl Piňos "Das Bekanntwerden" (1974, Urauff1976 Brno) für zwei Stimmen und Guittarre auf Texte von Zeitungsanzeigen gebaut. Der Autor verwendet hier sowie in anderen Kompositionen, einschließlich der elektronischen, eine rationelle Organisation des Musikmaterials.

Die Ausnuitzung der begrifflichen Seite der Sprache und die Wahl eines inhaltlich tragfähigen Textes findet ihren Gegensatz in der Ausnuitzung 
der klanglichen Seite der Sprache und in der Wahl vor allem klanglicher Texte. Auf diese Art gingen Autoren vor, die entweder für ihre Werke aus Fremdsprachen Wörter von derselben Bedeutung, wie z. B. Kopelent in "Snehah" (1966, Urauff-1966 Venezia, Druck-Supraphon 1967, Schpl-Supraphon 1970), oder umgekehrt einen inhaltlich bedeutenden Text in einer weniger bekannten Sprache wählen, so daß die Wirkung vor allem klanglich beschaffen ist. Das ist der Fall der Komposition "Caprichos" nach Goya von Luboš Fišer (1966, Urauff-1966 Praha, Druck-Supraphon 1967, Schpl-Supraphon 1970). Kopelents Snehah ist für Sopran, Jazz-Alt und Kammerensemble geschrieben. Die sinnreiche Komposition punktueller Art, die in der Aufführung den Raum sowie die Gegenüberstellung des lebendigen Klangs der Tonbandaufnahme ausnützt, ist im Wortteil auf den äquivalenten der "Liebe" in orientalen Sprachen gebaut. Durch die dreiteilige Form strebt dabei der Autor, die dreifache Deutung des berühmten altpersischen Vierzeilers Abu'sa'ida an. Caprichos von Fišer entstanden als ein Teil des Triptychons, das einen achttönigen Modus streng verarbeitet und in den zeitlichen Komponenten aleatorisch ist. Den Text der Komposition für Doppelchor à capella bildet die Wortbegleitung der Gra. phiken von Goyas Caprichos.

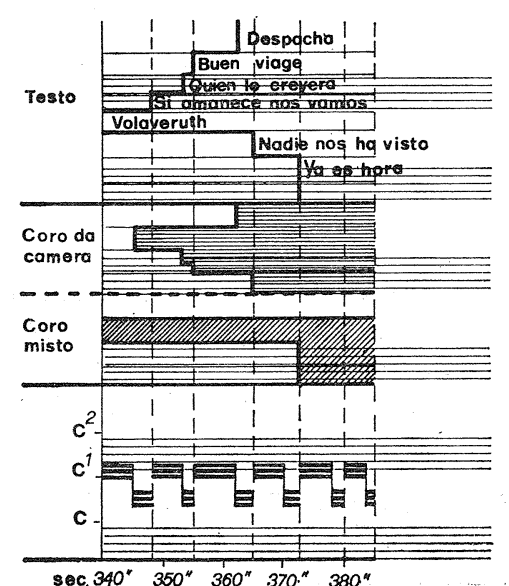

In diesem Zusammenhang ist auch die elektronische Komposition Jaroslav Krčeks "Sonáta slavíčková« (Nachtigall-Sonate) zu erwähnen (1969, Urauff-1970 Praha). Dieses Werk, in dem sich der Komponist auf die Artikulation der vokalen Äußerung konzentrierte, basiert auf einem Gedicht aus dem 17. Jh., das nur die Onomatopöie verwendet.

(Beide Tendenzen hatten ihre gefährlichen Extreme: am Rande der maximalen Ausnützung des Wortsinnes stand die Auflösung der Bindung zwischen dem Wort und der Musik und dadurch die Aufhebung der Gattung, und am Rande der entgegengesetzten Richtung, nämlich der Unterdrückung des Wortsinnes in der vokalen Äußerung, befand sich der Verlust des Sinnes. Diese Extreme zeigten sich jedoch nur als theoretisch.) 
Die Ausnützung der Sprache wird in der tschechischen vokalen Neuen Musik nicht nur an die Ausnützung ihrer begriffssemantischen oder klangsemantischen Qualitäten gebunden. Man benutzt die Sprache auch als Geste, Weisung, Verordnung oder Untersagung von einer symbolischen Art, und zwar dort, wo es sich um sinnreiche und allgemein bekannte Wörter einer Fremdsprache handelt. Auf diese Weise gingen Autoren vor, für denen Schaffen Integrationszüge typisch sind: Kabelác in "Eufemias Mysterion", Fišer in "Requiem" und Jan Tausinger in "Ave Maria». Das kriegfeindlich orientierte Werk Tausingers ist für Sopran, Rezitation und Orchester geschrieben, wobei es lateinischen Gesang mit tschechischer Rezita. tion konfrontiert (1973, Urauff-1972 Praha, Druck-Panton 1974, Schpl-Panton 1972). Fišers Requiem für Soli, Chor und Orchester (1968, Urauff-1968 Praha, Druck-Supraphon 1971) bildet den Schlußteil des Triptychons mit den »Caprichos" und "15 Blätter nach Dürers Apokalypse». In der Komposition wird mit demselben achttönigen Modus und Aleatorik in zeitlichen Komponenten wie in den vorhergehenden Werke gearbeitet.

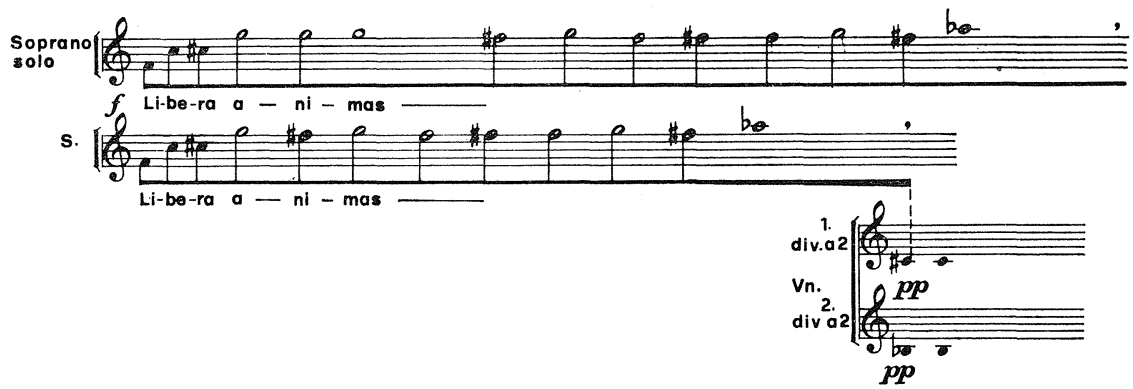

"Eufemias Mysterion« Kabeláčs für Sopran und Kammerorchester enstand im Jahre 1965 (Urauff-1965-Warszawa, Druck-SHV 1967, Schpl-Supraphon 1967). Der Sopranpart der vierteiligen Komposition benutzt griechische Wörter: Sige, eufemeite, eufemias mysterion, mit Hilfe deren er

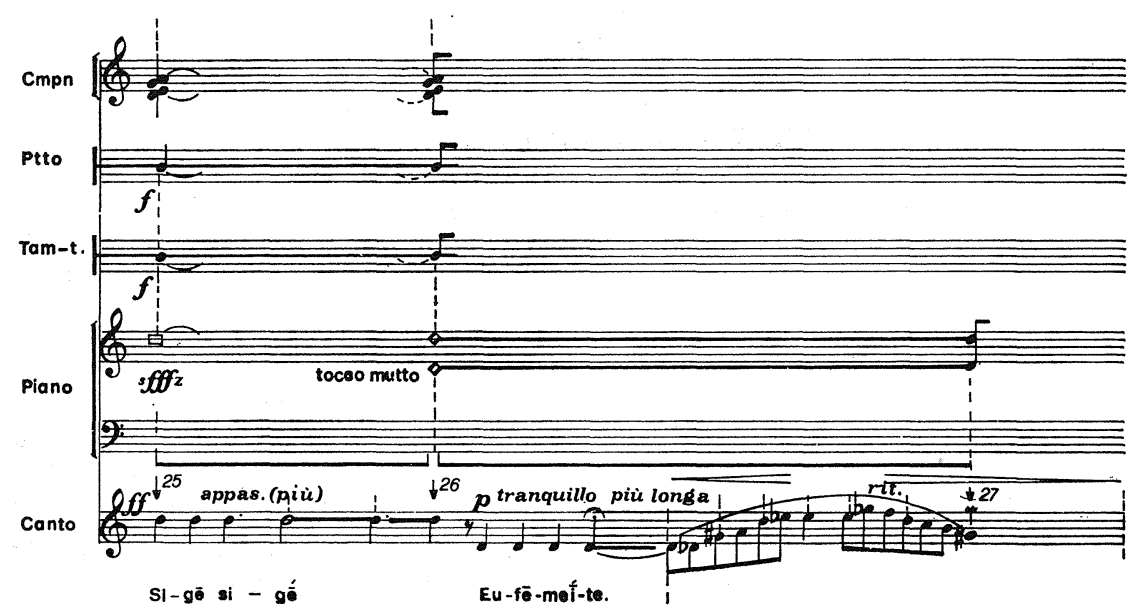


in der Gegenüberstellung dem Instrumentalklang eine symbolische konfliktbeladene Handlung mit Schlußkatharsis baut.

Mit einem anderen Sinn und auch anderen Kompositionsmethoden ist in der tschechischen Vokalmusik das Schaffen auf längere lateinische Texte verknüpft. Werke dieses Typus entstanden im Laufe der sechziger Jahre und wurden neben dem vorübergehenden Modecharakter durch die Anpassung der Textkomponente an neue Kompositionstechniken bedingt. Auf lateinischen Texte schrieben A. Simandl Piňos, M. Štědron̆, Z. Lukáš, I. Jirásek u. a. Im Unterschied zu den synthetisiereden Tendenzen, die für die oben erwähnte Gruppe von Werken typisch waren, ging es hier vielmehr um eine künstlerische Autonomie. Die Aufmerksamkeit wurde der Reinheit der kompositions-technischen Arbeit gewidmet, die allerdings die Kombi. nation verschiedener Techniken nicht ausschließt.

Andere Klangmöglichkeiten bot die elektronische Verarbeitung der vokalen Äußerung. Auf diese Art ging Zdeňek Lukáš in seiner Komposition "Ecce quomodo moritur justus" vor, die eine Motette von Jacobus Gallus verwendet (Urauff-1970 Praha). Das Werk ist gleichzeitig sowohl eine kunstvoll durchgeführte Analyse als auch eine Zitation des Originals, wobei das Vokalmaterial sich bis zu instrumentalen Klangelementen verwandelt. Auf ähnliche Weise geht Jan Klusák in der konkreten Motette "O sacrum convivium" vor, wenn er die melodische Linie einer zwei Stimmen im Unisono gesungenen Antiphone elektronisch verarbeitet (Urauff-1970 Praha).

Wenn einerseits die elektronische Verarbeitung des vokalen Materials ăhnlich wie die Ausnützung der instrumentalen Technik im vokalen Satz den vokalen Charakter der Vokalmusik abbauen kann, dann anderseits ist die aus der vokalen Äußerung hervorgehende Instrumentalmusik zu erwähnen. Hierher gehört auch das durch die städtische Folklore inspirierte Werk Václav Kučeras Argot (Urauff-1971 Praha), drei Sätze für Bläserquintett. Arnošt Parsch in seiner Musica concertante con omaggio (1970, Urauff-1971 Praha) kombiniert Zitationen aus Monteverdis Orfeo und Ritorno d'Ulisse mit eigener Musik, deren Material aus Monteverdis Musik abgeleitet ist.

Dieses Beispiel weist auf eine weitere Tendenz der Neuen Musik hin, die in der Unterstellung der Vokallinie neuen Kompositionstechniken, in der Ausnuitzung der doppelten Bedeutung der Sprache und in der Verknüpfung der Neuen Musik mit der Musik der Vergangenheit mittels der Zitation, Montage und Collage beruht. Diese Tendenz ist dadurch bedingt, daß die Neue Musik den Widerspruch der Sprache und der Musik in der Ebene des Materials nicht beseitigte, aber sie trug ihn nur in eine andere Ebene über: Die Unterstellung der Vokalmusik den neuen Kompositionsverfahren führte zu der Zerstückelung der Sprache und damit wurde die Bedeutungskonkretheit der Vokalmusik herabgesetzt. Diese Tatsache, u. a., führte zum Interesse für die Collage und ähnliche Techniken einerseits, und andererseits für außereuropäische musikalische Kulturen, d.h. zum Interesse für die Integrationsverfahren.

Unter diese Integrationsversuche reihen sich auch Bestrebungen, das vokale Werk mit dem visuellen $\mathrm{zu}$ verbinden. Vier Madrigale auf eigene 
Texte von V. Kučera aus dem Jahre 1975, betitelt Orbis pictus, wurden durch Werke von Václav Špála, Käthe Kollwitz, Paul Klee und Marc Chagall inspiriert und sie nützen verschiedene Elemente der menschlichen Stimme, einschließlich des Flüsterns, Aufschreis u. dgl. aus (Urauff-1975 Berlin). Breite Integrationstendenzen waren typisch für den tatkräftigen Brünner Team in ursprüngliche Zusammensetzung Berg-Piňos-štědron̆Růžička-Parsch. Seien hier besonders erwähnt team-work "Ecce homo« (Urauff-1969 Brno), Vokalsinfonie für Soli, Orchester und Tonband auf Worte aus dem Evangelium des hl. Johannes, dem Prozess und dem Schloß von Franz Kafka und aus den Sentenzen von Josef Berg, oder das Werk "Stimmliche Vernissage" (Urauff-1969 Brno) für Soli, sechs Instrumentalisten und zwei Speaker, das ein Spiel mit Textkombinationen verwendet und auf diese Weise in das Vokalwerk Theaterelemente eingliedert. Verschiedene Möglichkeiten, die auf die Konkretisierung der vokalen Komponente zielen, nützte V. Kučera in seiner Komposition "Lidice» (1972, Urauff1973 Praha, Schpl-Supraphon 1973) und L. Fišer in "Die Wahrheit von der Vernichtung der Stadt Ur« für Soli, Sprecher, Sänger- und Rezitationschöre, Glocken und Pauken auf ursumerischen Text aus (1970, Schpl-Panton 1972).

Auch die Beeinflußung durch außereuropäische Musikkulturen machte sich in der tschechischen Neuen Musik stark bemerkbar, jedoch vielmehr im instrumentalen als im vokalen Schaffen. Von solcherart orientierten Autoren sind Miloslav Ištván und besonders M. Kabeláč zu nennen. Die hier schon erwähnte 7. Sinfonie von Kabeláčs benutzt u.a. z. B. ein pentatonisches Klagelied aus der Neuen Guinea.

Die Kombination kompositorischer Techniken, Verflechtung der Vokalund Instrumentalmusik, Ausnützung der Zitation, Montage und Collage, sowie die Verbindung der Vokalmusik mit anderen Künsten müssen nicht unbedingt auf eine kunstvolle Synthese zielen, ähnlich wie das Bestreben nach einer originellen Kompositionstechnik nicht immer auf die künstlerische Autonomie gerichtet werden muß. Beide Grundtendenzen unterordnen sich jedoch vokale Äußerung und unterziehen sie ihren eigenen Gesetzmäßigkeiten. Deshalb ist die Tatsache, daß viele Autoren der tschechischen Neuen Musik für ihre Vokalkompositionen Texte in Fremdsprachen wählten, für die Gesamtorientierung der Neuen Musik wichtig, aber für die Aufgabe der Sprache in der Neuen Musik keineswegs maßgebend. Ebenfalls ist es bezeichnend, aber nicht von ersträngiger Bedeutung, daß viele Werke der Neuen Musik eine breite Registerskala der menschlichen Stimme einschließlich des Schreis, Lachens, Weinens, Flüsterns u. ä. benutzt. Wenn es auch scheint, daß für die tschechische vokale Neue Musik die Möglichkeiten, die Sprache als Baumaterial zu verwenden und verschiedene Intergrationsversuche zu verwirklichen, ausschlaggebend waren, kann man sagen, daß das Motiv der Entfaltung der tschechischen vokalen Neuen Musik weniger das Problem der Sprache und der Musik, als das Problem der Konfrontation der tschechischen Musik mit der Weltmusik war. 


\section{POVZETEK}

Nuva ceška vokalna glasba rešuje tako probleme, ki so skupni vsej novi glasbi kakor tudi nekatere specialne ceške; tako navezovanje na domačo tradicijo in komunikativno sposobnost ceškega jezika. Stanje nove čške vokalne glasbe šestdesetih let lahko karakteriziramo kot polarnost usmeritve $\mathrm{v}$ pojmovno semantično ali rodovno semantično kvaliteto jezika. V nadaljnjem razvoju nove glasbe so se uveljavili tehnika kolaža, uporaba elementov izvenevropskih glasbenih kultur in različni integracijski postopki (med novimi kompozicijskimi načini, vokalno in instrumentalno glasbo, med glasbo in drugimi umetnostmi). 